\title{
Epitaxial aluminum nitride tunnel barriers grown by nitridation with a plasma source
}

\author{
T. Zijlstra, C. F. J. Lodewijk, ${ }^{\text {a) }}$ N. Vercruyssen, F. D. Tichelaar, \\ D. N. Loudkov, and T. M. Klapwijk \\ Kavli Institute of Nanoscience, Faculty of Applied Sciences, Delft University of Technology, Lorentzweg 1, \\ 2628 CJ Delft, The Netherlands
}

(Received 26 June 2007; accepted 8 November 2007; published online 4 December 2007)

\begin{abstract}
High critical current-density (10 to $\left.420 \mathrm{kA} / \mathrm{cm}^{2}\right)$ superconductor-insulator-superconductor tunnel junctions with aluminum nitride barriers have been realized using a remote nitrogen plasma from an inductively coupled plasma source operated in a pressure range of $10^{-3}-10^{-1}$ mbar. We find a much better reproducibility and control compared to previous work. From the current-voltage characteristics and cross-sectional transmission electron microscopy images it is inferred that, compared to the commonly used $\mathrm{AlO}_{x}$ barriers, the polycrystalline AlN barriers are much more uniform in transmissivity, leading to a better quality at high critical current densities. (c) 2007 American Institute of Physics. [DOI: 10.1063/1.2819532]
\end{abstract}

Quantum technology based on superconducting or magnetic metals uses nanometer thick tunnel barriers. Many routes to quantum computation are based on aluminum with aluminum oxide barriers. Niobium devices use a proximitized layer of aluminum with a similar oxide. ${ }^{1}$ Magnetic tunnel junctions have recently moved from using amorphous aluminum oxide to epitaxial magnesium oxide with its unique spin-dependent properties. ${ }^{2,3}$ In quantum computation the amorphous tunnel barrier has surfaced as an important source of decoherence leading to the introduction of an epitaxial aluminum oxide barrier. ${ }^{4,5}$ On the other hand, highly transmissive tunnel barriers are urgently needed for submillimeter mixers in order to achieve a high bandwidth, ${ }^{6}$ in electronic refrigeration to maximize the cooling power, ${ }^{7}$ and in high density magnetic memory devices. ${ }^{8}$

It has been demonstrated that a major problem of amorphous $\mathrm{AlO}_{x}$ barriers is that they are laterally inhomogeneous. ${ }^{9,10}$ We take this into account by using a distribution of transparencies $T_{n}$ by writing for the voltageindependent normal conductance,

$$
G \propto \sum_{n} A_{n} T_{n},
$$

with $A_{n}$ a fraction of the total area of the tunnel barrier with an assumed uniform transmissivity $T_{n}$. Hence, we do not assume a universal distribution of transparencies ${ }^{11,12}$ but one which is related to a distribution of areas with different transmissivities, resulting from the technological process. For superconducting tunnel junctions (SIS) this amounts to a situation analogous to superconducting quantum point contacts, ${ }^{13}$

$$
I \propto \sum_{n} A_{n} j\left(V, T_{n}\right),
$$

with $I$ the total current and $j\left(V, T_{n}\right)$ the voltage-dependent current density per area $A_{n}$. $j$ contains contributions of different orders proportional to $T_{n}, T_{n}^{2}, T_{n}^{3}$, etc., reflecting multiple Andreev reflections $\left[j\left(V, T_{n}\right)=j_{1}\left(V, T_{n}\right)+j_{2}\left(V, T_{n}^{2}\right)\right.$ $\left.+j_{3}\left(V, T_{n}^{3}\right)+\cdots\right]$. For the commonly used low current-density

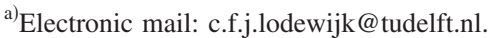

tunnel barriers, most $A_{n}$ have $T_{n}$ of the order of $10^{-4}$. Since for SIS junctions first order tunneling $j_{1}$ leads to a zero subgap current, the remaining subgap current is due to the higher order terms $\left(j_{2}, j_{3}, \ldots\right)$, which only appear for $T_{n} \approx 1$. Nonuniformity, causing the emergence of areas with $T_{n} \approx 1$, thus leads to excessive subgap currents. Therefore, the critical current density of amorphous aluminum oxide barriers is limited to $20 \mathrm{kA} / \mathrm{cm}^{2} .{ }^{14}$ We will demonstrate that aluminum nitride barriers are superior to aluminum oxide barriers with respect to barrier uniformity.

In the work reported here a very good reproducibility is realized by using the afterglow region of a nitrogen plasma from an inductively coupled plasma source (COPRA) (see, for example, Ref. 15), from CCR Technology. The plasma provides the energy to split the $\mathrm{N}_{2}$ molecules into $\mathrm{N}$ radicals, needed for the growth of AlN. The source is mounted on a vacuum chamber. The plasma is created in the source and diffuses into the chamber. We have chosen to work in a range of high pressures $\left(2 \times 10^{-3}-1 \times 10^{-1} \mathrm{mbar}\right)$, for two reasons. First, we expect at these higher pressures a larger fraction of atomic N. Secondly, the ion energies in this regime are as low as a few eV, which minimizes damage to the barrier formation. This is different from recent work, where the plasma process not only provides the chemically active species but also creates damage by highly energetic ions, ${ }^{16-20}$ although usable routes have been reported. ${ }^{18}$ In addition, many other plasma techniques suffer from instabilities, resulting in a poor process reproducibility.

The devices are fabricated on a 2 in. oxidized silicon or fused quartz substrate. All metal layers are deposited by magnetron sputtering in the process chamber of a Kurt Lesker system. First, a $100 \mathrm{~nm}$ Nb monitor layer is deposited, after which a ground plane pattern is optically defined. Subsequently, a bilayer of $100 \mathrm{~nm} \mathrm{Nb}$ and about $7 \mathrm{~nm} \mathrm{Al}$ is deposited. Without breaking the vacuum, the substrate is then transferred to a nitridation chamber, where the $\mathrm{Al}$ is exposed to the nitrogen plasma for several minutes, producing a layer of AlN. The substrate is then again in vacuo transferred to the process chamber, where a top electrode of $200 \mathrm{~nm} \mathrm{Nb}$ is deposited. The lateral dimensions of the multilayer of $\mathrm{Nb} / \mathrm{Al} / \mathrm{AlN} / \mathrm{Nb}$ are patterned by lift-off. Junc- 


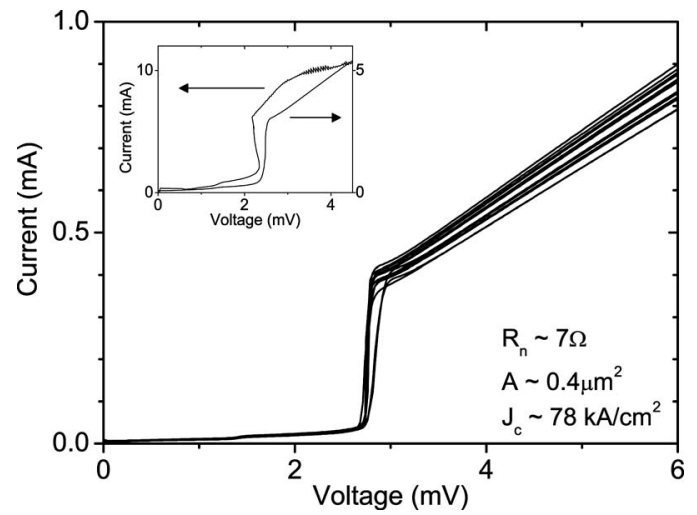

FIG. 1. Current-voltage characteristics of a typical batch of $\mathrm{Nb} / \mathrm{AlN} / \mathrm{Nb}$ junctions. Junction area is about $0.4 \mu \mathrm{m}^{2}$, for a normal resistance $R_{n}$ of $6.8 \Omega$ (critical current density $\sim 78 \mathrm{kA} / \mathrm{cm}^{2}$ ). The Josephson current has been suppressed with a magnetic field. Inset shows $I V$ characteristics of SIS junctions with $J_{c}$ of 130 and $420 \mathrm{kA} / \mathrm{cm}^{2}$. For the latter thermal heating causes gap suppression and back bending.

tions are defined by e-beam lithography with a negative e-beam resist (SAL-601) layer and reactively ion etched (RIE) with a $\mathrm{SF}_{6} / \mathrm{O}_{2}$ plasma using the AlN as an etch stop, followed by a mild anodization $(5 \mathrm{~V})$. The junction resist pattern is used as a self-aligned lift-off mask for a dielectric layer of $250 \mathrm{~nm} \mathrm{SiO}$. A $500 \mathrm{~nm} \mathrm{Nb} / 50 \mathrm{~nm} \mathrm{Au}$ top layer is deposited and $\mathrm{Au}$ is etched with a wet etch in a $\mathrm{KI} / \mathrm{I}_{2}$ solution using an optically defined mask. Finally, using an e-beam defined top wire mask pattern, the layer of $\mathrm{Nb}$ is etched with a $\mathrm{SF}_{6} / \mathrm{O}_{2} \mathrm{RIE}$, which finishes the fabrication process.

The fabrication process used provides a very good reproducibility. There is reproducibility within one fabrication run, illustrated by the similarity of junctions on a produced wafer. Scatter in the normal resistance $R_{n}$ of the junctions is caused by variation in the junction area $A$, due to uncertainties in junction definition and variation in the barrier-specific $R_{n} A$ value. For 34 junctions, of which 14 are shown in Fig. 1, the standard deviation, $\sqrt{\sum_{i=1}^{m}\left(R_{n, i}-\left\langle R_{n}\right\rangle\right)^{2} /(m-1)}$, with $m$ the number of junctions, of $R_{n}$ has been determined to be $3.3 \%$ relative to the average, $\left\langle R_{n}\right\rangle=6.8 \Omega$. The peak-to-peak variation amounts to $\pm 6 \%$. By measuring four big junctions (two of $1 \mu \mathrm{m}^{2}$ and two of $2 \mu \mathrm{m}^{2}$ ), the value of $R_{n} A$ has been found to be $2.8 \Omega \mu \mathrm{m}^{2}$ (corresponding to a critical current density $J_{c} \approx 78 \mathrm{kA} / \mathrm{cm}^{2}$ ). Assuming perfect junction definition (which is most likely not the case), the standard deviation of $R_{n} A$ within one fabrication run is at most $3.3 \%$. Based on the average $R_{n}, A$ is $0.4 \mu \mathrm{m}^{2}$.

We also find a good reproducibility from run to run. We have made several batches, varying the nitridation time $t_{N}$ from 9 to $60 \mathrm{~min}$. About half of the batches have been made with a low position of the chuck $(30 \mathrm{~cm}$ distance to the plasma source) in the nitridation chamber, the other half with a higher position (15 cm distance to the plasma source). In Fig. 2, we plot the $R_{n} A$ product of the batches as a function of $t_{N}$ for the large chuck-source distance (squares) and for the small chuck-source distance (diamonds). The dashed lines indicate a dependence $R_{n} A \propto t_{N}^{k}$, with $k=1.4$. Obviously, there is a systematic dependence on nitridation time, indicating a well-behaving process. By varying the nitridation time and/or the chuck position, we can realize any desired $R_{n} A$ value between 0.5 and $10 \Omega \mu \mathrm{m}^{2}$. value between 0.5 and $10 \Omega \mu \mathrm{m}^{2}$ shows significantly better reproducibility than other AlN
Downloaded 10 Aug 2010 to 131.180 .130 .114$. Redistribution subject to AIP license or copyright; see http://apl.aip.org/apl/copyright.jsp

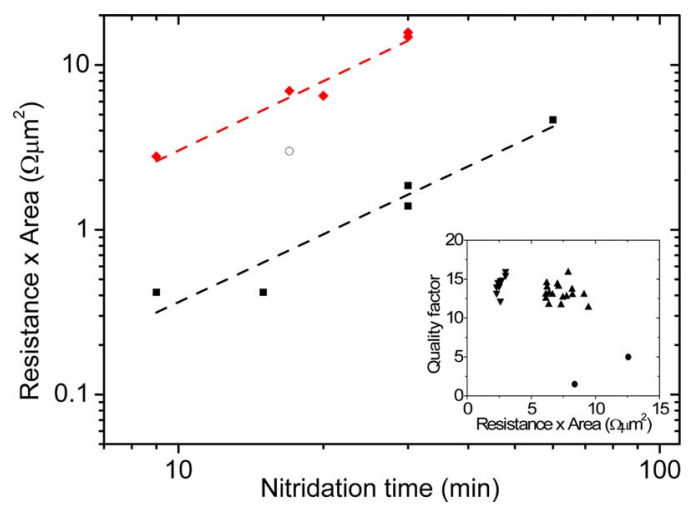

FIG. 2. (Color online) $R_{n} A$ product as a function of nitridation time $t_{N}$ for nine different fabricated batches. The squares represent data for a $30 \mathrm{~cm}$ chuck-source distance, whereas the diamonds indicate a $15 \mathrm{~cm}$ chuck-source distance. The open circle represents the junctions of Fig. 1. Dashed lines indicate both a dependence $R_{n} A \propto t_{N}^{1.4}$. Inset: Quality factor as a function of $R_{n} A$ product for two batches of $\mathrm{Nb} / \mathrm{AlN} / \mathrm{Nb}$ junctions (up- and downpointing triangles). Also indicated are $\mathrm{AlO}_{x}$ data from Miller et al. (Ref. 14) (filled circles).

The quality factor $Q$, defined as $R_{\mathrm{sg}} / R_{n}$, where $R_{\mathrm{sg}}$ is the subgap resistance, gives an indication of the subgap leakage through the tunnel barrier. In the inset of Fig. 2, $Q$ has been plotted for two different batches of AlN based junctions, together with data on $\mathrm{AlO}_{x}$ from Miller et al. ${ }^{14}$ In contrast to these $\mathrm{AlO}_{x}$ devices, it is evident that $Q$ is higher than 10 for all AlN devices. The lower subgap currents prove that the AlN barriers have a lower density of areas with $T_{n} \approx 1$, in other words a better uniformity.

As shown in Fig. 2, we reach $R_{n} A$ products as low as $0.4 \Omega \mu \mathrm{m}^{2}$, corresponding to a $J_{c}$ of $420 \mathrm{kA} / \mathrm{cm}^{2}$. For such high current densities, heating effects decrease the superconducting gap voltage of the junction in the form of back bending (Fig. 1, inset). Up to at least $130 \mathrm{kA} / \mathrm{cm}^{2}$, this effect remains hidden but is still present. This indicates that for these AlN barriers the maximum critical current density is no longer limited by the materials control of the barrier, but by the intrinsic physical process of nonequilibrium in the electrodes. Consequently, a future detailed statistical evaluation of reproducibility and control has to take into account the distortions of the $I V$ curves by heating.

Using high-resolution transmission electron microscopy (HRTEM), we find that the AlN barrier is grown epitaxially (Fig. 3). The barrier is visible as a region with higher transmission (most bright region) and in a small difference in lattice spacing for $\mathrm{Al}$ and $\mathrm{AlN}$. The images clearly indicate epitaxial alignment of the crystalline structure of the AlN with the underlying Al crystal. The lattice plane distances of the planes parallel to the surface were measured in various locations and identified as either $\{0002\}$ or $\{1 \overline{101}\}$ planes in the hexagonal AlN phase, with spacings of $2.49 \pm 0.06$ and $2.37 \pm 0.06 \AA$, respectively. An averaged thickness of the barrier of about $1.5 \pm 0.5 \mathrm{~nm}$ is found. For these devices the $R_{n} A$ value is about $16 \Omega \mu \mathrm{m}^{2}$. Obviously, in contrast to the commonly used $\mathrm{AlO}_{x}$, the AlN tunnel barrier has a crystalline nature with a thickness of about six lattice planes, which may be the cause of the better uniformity.

In conclusion, epitaxial aluminum nitride tunnel barriers have been grown, at ambient temperature, using a plasma source to generate chemically active nitrogen. This method 


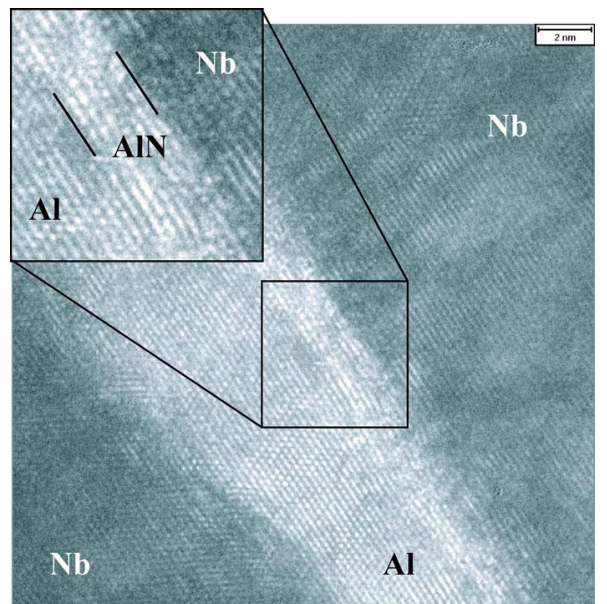

FIG. 3. (Color online) HRTEM micrographs of an AlN dielectric barrier deposited on an Al layer (bright region) between $\mathrm{Nb}$ electrodes (dark regions). The bar in the top-right corner represents a length of $2 \mathrm{~nm}$.

growth techniques have shown in the past. Compared to the conventionally used aluminum oxide barriers, much better quality current-voltage characteristics are observed for high critical current densities, which is attributed to a spatially more uniform transmissivity of the epitaxial tunnel barrier.

The authors would like to thank B. de Lange for technical assistance, and P. C. Snijders and R. W. Simmonds for discussions. We thank NanoImpuls, Nanofridge, the Dutch Research School for Astronomy (NOVA), the Dutch Organisation for Scientific Research (NWO), and the European Southern Observatory (ESO) for funding this project.
${ }^{1}$ M. Gurvitch, M. A. Washington, and H. A. Huggins, Appl. Phys. Lett. 42, 472 (1983).

${ }^{2}$ S. S. P. Parkin, C. Kaiser, A. Panchula, P. M. Rice, B. Hughes, M. Samant, and S.-H. Yang, Nat. Mater. 3, 862 (2004)

${ }^{3}$ S. Yuasa, T. Nagahama, A. Fukushima, Y. Suzuki, and K. Ando, Nat. Mater. 3, 868 (2004).

${ }^{4}$ S. Oh, K. Cicak, R. McDermott, K. B. Cooper, K. D. Osborn, R. W. Simmonds, M. Steffen, and D. P. Pappas, Solid State Commun. 18, 1396 (2005).

${ }^{5}$ S. Oh, K. Cicak, J. S. Kline, M. A. Sillanpää, K. D. Osborn, J. D. Whittaker, R. W. Simmonds, and D. P. Pappas, Phys. Rev. B 74, 100502 (2006).

${ }^{6}$ J. Kawamura, D. Miller, J. Chen, J. Zmuidzinas, B. Bumble, H. G. LeDuc, and J. A. Stern, Appl. Phys. Lett. 76, 2119 (2000).

${ }^{7}$ F. Giazotto, T. T. Heikkila, A. Luukanen, A. M. Savin, and J. P. Pekola, Rev. Mod. Phys. 78, 217 (2006).

${ }^{8}$ K. Tsunekawa, D. D. Djayaprawira, S. Yuasa, M. Nagai, H. Maehara, S. Yamagata, E. Okada, N. Watanabe, Y. Suzuki, and K. Ando, IEEE Trans. Magn. 42, 103 (2006).

${ }^{9}$ W. H. Rippard, A. C. Perrella, F. J. Albert, and R. A. Buhrman, Phys. Rev. Lett. 88, 046805 (2002).

${ }^{10}$ K. M. Lang, D. A. Hite, R. W. Simmonds, R. McDermott, D. P. Pappas, and J. M. Martinis, Rev. Sci. Instrum. 75, 2726 (2004).

${ }^{11}$ K. M. Schep and G. E. W. Bauer, Phys. Rev. Lett. 78, 3015 (1997).

${ }^{12}$ Y. Naveh, V. Patel, D. V. Averin, K. K. Likharev, and J. E. Lukens, Phys. Rev. Lett. 85, 5404 (2000).

${ }^{13}$ E. Scheer, P. Joyez, D. Esteve, C. Urbina, and M. H. Devoret, Phys. Rev. Lett. 78, 3535 (1997).

${ }^{14}$ R. E. Miller, W. H. Mallison, A. W. Kleinsasser, K. A. Delin, and E. M. Macedo, Appl. Phys. Lett. 63, 1423 (1993).

${ }^{15}$ M. Weiler, K. Lang, E. Li, and J. Robertson, Appl. Phys. Lett. 72, 1314 (1998).

${ }^{16}$ T. Shiota, T. Imamura, and S. Hasuo, Appl. Phys. Lett. 61, 1228 (1992).

${ }^{17}$ Z. Wang, A. Kawakami, and Y. Uzawa, Appl. Phys. Lett. 70, 114 (1997).

${ }^{18}$ B. Bumble, H. G. LeDuc, J. A. Stern, and K. G. Megerian, IEEE Trans. Appl. Supercond. 11, 76 (2001).

${ }^{19}$ A. B. Kaul, A. W. Kleinsasser, B. Bumble, H. G. LeDuc, and K. A. Lee, J. Mater. Res. 20, 3047 (2005).

${ }^{20}$ N. N. Iosad, A. B. Ermakov, F. E. Meijer, B. D. Jackson, and T. M. Klapwijk, Supercond. Sci. Technol. 15, 945 (2002). 